\title{
Flora vascular não arbórea de um reflorestamento implantado com espécies nativas
}

\author{
Michel Anderson Almeida Colmanetti ${ }^{1,3}$, Regina Tomoko Shirasuna ${ }^{2}$ e Luiz Mauro Barbosa ${ }^{2}$
}

Recebido: 7.05.2015; aceito: 1.10 .2015

\begin{abstract}
Non-arboreal vascular flora in a reforestation implanted with native seedlings). Non-arboreal life forms, such as herbs, vines, and subshrubs, have rarely been assessed in monitoring of reforestations that aim at restoration ecology. This study aimed to assess the non-arboreal species from a reforestation implanted with a composition of Seasonal Semi-deciduous Forest, nine years after the plantation. The results showed high number of non-arboreal species, from which, the ones established by natural regeneration, had more species than the tree layer. We found one rare and one critically endangered species that revealed the importance of non-arboreal flora for the conservation of biodiversity and as a potential indicator for monitoring in restoration process.
\end{abstract}

Keywords: natural regeneration, restoration ecology, RPPN

RESUMO - (Flora vascular não arbórea de um reflorestamento implantado com espécies nativas). Formas de vida não arbóreas, como ervas, trepadeiras e subarbustos, raramente são avaliadas em estudos de monitoramento de reflorestamentos que visam à restauração ecológica. O objetivo do presente estudo foi inventariar as espécies não arbóreas em um reflorestamento implantado com composição de Floresta Estacional Semidecídua, nove anos após o plantio. Os resultados obtidos revelaram elevado número de espécies não arbóreas, das quais as estabelecidas por regeneração natural, que foram registradas em maior número de espécies do que aquelas do estrato arbóreo. Foi encontrada uma espécie rara e uma criticamente ameaçada, destacando a importância dessa vegetação para a conservação da biodiversidade e como um potencial indicador para monitoramento de áreas em processo de restauração.

Palavras-chave: regeneração natural, restauração ecológica, RPPN

\section{Introdução}

Diferentes indicadores têm sido utilizados para monitorar áreas em processo de restauração, com a finalidade de verificar se os objetivos esperados foram atingidos. Sabe-se que os indicadores devem estar de acordo com a idade dos reflorestamentos, considerando variáveis como a diversidade e riqueza de espécies, área basal, altura média, chuva e banco de sementes, estrato regenerante, cobertura de copa, presença de espécies invasoras, presença de fauna, entre vários outros (Siqueira 2002, Sorreano 2002, Souza \& Batista 2004, Barbosa \& Piso 2006, Damasceno 2005, Melo et al. 2007, Bellotto et al. 2009, Suganuma et al. 2008, Silveira et al. 2011, Trevelin et al. 2013).

Quando se trata da vegetação, a maioria dos estudos está voltada para as espécies arbóreas que foram plantadas ou que surgiram por regeneração natural. Para o Estado de São Paulo essa tendência é pertinente, considerando que a atual orientação (Resolução SMA 32/2014) contempla, com maior ênfase, a vegetação arbórea. Embora seja discutível uma maior abrangência, incluindo espécies que não sejam apenas arbóreas em projetos de restauração (Durigan et al. 2010), ainda não há nenhuma proposta para se contemplar essas outras formas de crescimento em documentos formais de políticas públicas.

Apesar de existirem alguns estudos envolvendo essas espécies em remanescentes florestais (Rossato et al. 2008, Menini Neto et al. 2009, Oliveira 2012, Dinato 2014), esses ainda são escassos em áreas em processo de restauração ecológica (Garcia 2012, Duarte \& Gandolfi 2013), havendo várias lacunas que devem ser esclarecidas, como a idade adequada para se realizar realocação de espécies epífitas em reflorestamentos (Duarte \& Gandolfi 2013).

Para florestas secundárias, sabe-se que a comunidade de epífitas é mais abundante no estágio

1. Universidade de São Paulo, Escola Superior de Agricultura "Luiz de Queiroz", 13418-260 Piracicaba, SP, Brasil

2. Instituto de Botânica de São Paulo, Coordenação Especial de Restauração Ecológica, 04301-902 São Paulo, SP, Brasil

3. Autor para correspondência: michelcolmanetti@gmail.com 
avançado, e que algumas espécies de gramíneas, ervas e pteridófitas podem ocorrer no processo inicial de colonização da área (Guariguata \& Ostertag 2001). Quando se trata de reflorestamentos, ainda são raros os estudos que visam a elucidar se os sistemas de plantios utilizados são suficientes para subsidiar a regeneração natural de espécies não arbóreas (Garcia 2012). É necessário verificar a ocorrência dessas espécies em áreas reflorestadas, a fim de observar se o estrato arbóreo formado pelas espécies plantadas pode criar um habitat que favoreça o estabelecimento das espécies não arbóreas, com alterações no microclima, luz, entre outros, conforme verificado para florestas secundárias (Guariguata \& Ostertag 2001).

O objetivo deste estudo é conhecer riqueza de espécies não arbóreas, que incluem trepadeiras, subarbustos, ervas terrícolas e epífitas, em um reflorestamento nove anos após o plantio. A importância desses componentes para a conservação da biodiversidade é abordada e enfatizada como possível indicador para monitoramento em restauração ecológica.

\section{Material e métodos}

O presente estudo foi realizado na Reserva Particular do Patrimônio Natural (RPPN) Parque Florestal São Marcelo, pertencente à empresa International Paper do Brasil LTDA. A RPPN reconhecida legalmente consiste em 240 ha, formada a partir de um reflorestamento com espécies nativas realizado em 2002. No entanto, a área de estudo, compreende parte da RPPN, aproximadamente 180 ha (figura 1). A vegetação da região é composta de Floresta Estacional Semidecídua (Aquino \& Barbosa 2009), porém também é formada por Cerrado, com variações de Cerradão a Campo Cerrado. O clima da região é classificado como $\mathrm{C}_{\mathrm{wa}}$, temperado quente com estiagem no inverno (Köppen 1948).

Foram plantadas 100 espécies nativas de Floresta Estacional Semidecídua, com uma proporção de $56 \%$ de não pioneiras, $37 \%$ de pioneiras, e $7 \%$ de espécies não classificadas. Não há informação sobre a proporção de indivíduos por espécie, porém, sabe-se que não ultrapassou $20 \%$ de indivíduos de uma mesma espécie. A distribuição das espécies no momento do plantio ocorreu ao acaso, sendo alternadas nas linhas de espécies pioneiras e não pioneiras. Utilizou-se espaçamento de $3,0 \times 2,5 \mathrm{~m}$ e densidade de 1.333 mudas ha-1.

No passado a propriedade era destinada ao plantio de culturas de ciclo anual, semiperenes, como cana-de-açúcar; e perenes, como café, citros, além de pastagem. Após a aquisição da área pela International Paper, em 1995, a área foi destinada unicamente ao plantio de Eucalyptus sp., até o ano de 2002. Nesse mesmo ano, 240 ha foram destinados à implantação da RPPN. A RPPN reconhecida legalmente, somada a outros reflorestamentos com espécies nativas e aos remanescentes florestais presentes na área, forma um contínuo de vegetação, totalizando 536 ha.

As áreas circunvizinhas à RPPN são compostas por propriedades rurais, incluindo o Horto MogiGuaçu pertencente à Empresa, destinado ao plantio comercial de Eucalyptus sp., existindo pequenos fragmentos florestais representados por faixas ciliares de vegetação nativa remanescente. As matas ciliares dos rios Mogi-Guaçu e Mogi-Mirim, que se encontram dentro da propriedade, foram implantadas entre 1996 e 1998, com baixa diversidade específica (cerca de 30 espécies arbóreas); e o fragmento florestal com maior expressão está localizado numa propriedade vizinha, pertencente a terceiros, a uma distância aproximada de $10 \mathrm{~km}$ da RPPN. Melhores descrições sobre o plantio e reflorestamento constam em Colmanetti (2013).

O levantamento florístico das trepadeiras, subarbustos, ervas terrícolas e epífitas foi realizado dentro da área de abrangência da RPPN, durante o período de 2011 a 2012, quando a área apresentava entre nove e 10 anos. Na RPPN existem 40 parcelas permanentes, das quais $20(12,4 \times 18 \mathrm{~m})$ vêm sendo monitoradas há mais de uma década, com enfoque

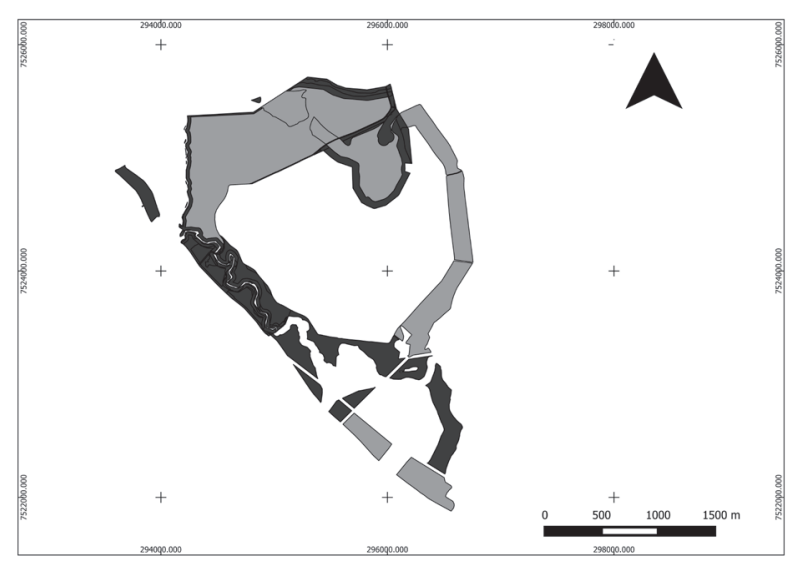

Figura 1. Reflorestamento da RPPN, Parque Florestal São Marcelo e vegetação remanescente de propriedade da International Paper, em Mogi-Guaçu, SP, Brasil. $\square$ RPPN - Reconhecida, Demais Áreas (Vegetação)

Figure 1. Reforestation of Parque Florestal São Marcelo RPPN and remnants of vegetation in Mogi-Guaçu owned by International Paper, São Paulo State, Brazil. $\square$ RPPN - Legally recognized, Other areas (Vegetation). 
na vegetação arbórea. No presente estudo, foram identificadas todas as espécies não arbóreas dentro e nas proximidades das 20 parcelas amostrais com nove anos após o plantio.

Foram identificadas todas as espécies não arbóreas dentro das 20 parcelas amostrais. Tendo as parcelas amostrais como ponto de origem, utilizou-se o método de caminhamento para coleta das espécies fora das parcelas amostrais, segundo Figueiras et al. (1994). Foram coletadas e identificadas todas as espécies não arbóreas que incluem as ervas terrícolas, ervas epífitas, trepadeiras e subarbustos. Foram realizadas coletas mensais, com três dias de duração, de fevereiro de 2011 a fevereiro de 2012 (13 expedições). A identificação das espécies ocorreu in loco e aquelas que não puderem ser identificadas desta forma, foram herborizadas e encaminhadas ao Herbário do Instituto de Botânica de São Paulo, para identificação por meio de consulta a especialistas. A listagem foi organizada de acordo com o Angiosperm Phylogeny Group (APG III 2009) e Smith et al. (2006). A nomenclatura foi atualizada e confirmada segundo a Lista de Espécies da Flora do Brasil (2015) e na base dados online Tropicos, do Missouri Botanical Garden (MOBOT 2015). Os espécimes foram incorporados no acervo do Herbário SP (Instituto de Botânica de São Paulo), seguindo técnicas tradicionais (Mori et al. 1985).

Para classificação da forma de vida e substrato foi utilizada a Flora do Brasil (2015), sendo considerados os termos: ervas, trepadeiras e subarbustos. Destaca-se que lianas e vinhas foram classificadas apenas como trepadeiras. Quanto ao substrato, consideraram-se hemi-hepífitas as plantas que iniciam seu ciclo de vida como epífitas e emitem raízes adventícias até o solo, tornando-se, depois, plantas terrestres; e epífitas as plantas que vivem sobre outras, utilizando-as como suporte, sem parasitá-las (IBGE, 2012). Consideraram-se terrícolas aquelas espécies que se desenvolvem e crescem sobre o solo.

Quanto à origem dos táxons, seguiu-se a Flora do Brasil (2015), utilizando as definições de acordo com Moro et al. (2012) que considerou: exóticas aquelas espécies que não ocorreriam naturalmente em uma dada região geográfica sem o transporte humano (intencional ou acidentalmente) para a nova região; naturalizadas como aquelas exóticas que conseguem se reproduzir de modo consistente no local onde foram introduzidas, de modo a estabelecer uma população autoperpetuante sem a necessidade da intervenção humana direta, mas que, entretanto, não se dispersam para longe do local de introdução; e invasoras as exóticas que, além de conseguir reproduzir-se consistentemente e manter uma população viável autonomamente, também conseguem dispersar-se para áreas distantes do local original da introdução e lá estabelecer-se, invadindo a nova região geográfica para onde foram levadas. Foram consideradas espécies invasoras aquelas classificadas por Zenni \& Ziller (2011).

\section{Resultados e Discussão}

Foram registradas 35 famílias, 85 gêneros e 110 espécies não arbóreas, sendo 88 espécies nativas (tabela 1). Entre as pteridófitas, foram identificadas sete famílias, 10 gêneros e 15 espécies, das quais duas espécies foram exóticas. Nota-se o predomínio de ervas de hábito terrícola. Destaca-se a presença da espécie naturalizada Pellaea viridis (Forssk.) Prantl (sinônimo heterotípico de Pellaea flavescens Fée, antes considerada rara e revista por Prado (2015)), sendo essa a segunda ocorrência da espécie para o Estado de São Paulo (Prado \& Hirai 2011). Já em relação às fanerógamas, foram encontradas 95 espécies, destacando-se a presença de uma criticamente ameaçada, Oxalis cratensis Oliv. ex Hook. (Mamede et al. 2007).

Colmanetti (2013) verificou, nas mesmas subparcelas do presente estudo, 86 espécies arbóreas. Estas espécies, somadas às não arbóreas encontradas neste estudo, correspondem a uma riqueza total de 196 espécies. Destaca-se que a RPPN inicialmente foi implantada apenas com espécies arbóreas, porém, nove anos após o plantio, as espécies predominantes não são arbóreas (56\%), oriundas de regeneração natural (figura 2). Já quando se trata apenas de espécies nativas, a riqueza da RPPN chegou a 164, destas 53\% não arbóreas.

Em Florestas Semidecíduas a riqueza de espécies não arbóreas pode facilmente chegar a 200 espécies (Oliveira 2012, Dinato 2014). No entanto, há poucos estudos voltados para a vegetação não arbórea em áreas reflorestadas. Garcia (2012) verificou 53 e 58 espécies de arbustos, subarbustos, ervas epífitas, e trepadeiras, respectivamente, em reflorestamentos com 12 e 50 anos após o plantio. Já Damasceno (2005) constatou a presença de apenas 20 espécies de trepadeiras e apenas uma espécie de epífita em reflorestamentos heterogêneos com idades iguais ou inferiores a 16 anos.

O grau de intervenção antrópica é um fator determinante no número de espécies epífitas, sendo 
Tabela 1. Espécies não arbóreas ocorrentes na RPPN Parque São Marcelo, Mogi Guaçu, SP, Brasil. * Espécie rara (segunda citação para o Estado de São Paulo). ** Em perigo crítico (Mamede et al. 2007).

Table 1. Non-arboreal species present at Parque São Marcelo RPPN, Mogi Guaçu, São Paulo State, Brazil. * Rare species (second citation to São Paulo State).** Critically endangered (Mamede et al. 2007).

\begin{tabular}{|c|c|c|c|}
\hline Espécie & Forma de vida/substrato & Origem & Voucher \\
\hline \multicolumn{4}{|l|}{ Pteridófitas } \\
\hline \multicolumn{4}{|l|}{ Anemiaceae } \\
\hline Anemia phyllitidis (L.) Sw. & Erva/Terrícola & Nativa & Shirasuna 2966 \\
\hline Anemia radiana link & Erva/Terrícola & Nativa & Shirasuna 2964 \\
\hline \multicolumn{4}{|l|}{ Blechnaceae } \\
\hline Blechnum occidentale L. & Erva/Terrícola & Nativa & Shirasuna 2962 \\
\hline \multicolumn{4}{|l|}{ Doryopteridaceae } \\
\hline Doryopteris cf. concolor (Langsd. \& Fisch.) J.Sm. & Erva/Terrícola & Nativa & \\
\hline \multicolumn{4}{|l|}{ Ophioglossaceae } \\
\hline Ophioglossum reticulatum $\mathrm{L}$. & Erva/Terrícola & Nativa & \\
\hline \multicolumn{4}{|l|}{ Polypodiaceae } \\
\hline Microgramma squamulosa (Kaulf.) de la Sota & Erva/Epífita & Nativa & Shirasuna 3536 \\
\hline Pleopeltis angusta Humb. \& Bonpl. ex Willd. & Erva/Epífita & Exótica & \\
\hline Pleopeltis hirsutissima (Raddi) de la Sota & Erva/Epífita & Nativa & Shirasuna 2949 \\
\hline Pleopeltis pleopeltifolia (Raddi) Alston & Erva/Epífita & Nativa & Shirasuna 2953 \\
\hline \multicolumn{4}{|l|}{ Pteridaceae } \\
\hline Doryopteris concolor (Langsd. \& Fisch.) J. Sm. & Erva/Terrícola & Nativa & Shirasuna 2952 \\
\hline Doryopteris nobilis (T.Moore) C. Chr. & Erva/Terrícola & Nativa & Shirasuna 2968 \\
\hline Doryopteris pedata (L.) Fée & Erva/Terrícola & Exótica & \\
\hline Pellaea viridis (Forssk.) Prantl & Erva/Terrícola & Naturalizada & Shirasuna 3133 \\
\hline Pteris multifida Poir. & Erva/Terrícola & Naturalizada & Shirasuna 2967 \\
\hline \multicolumn{4}{|l|}{ Thelypteridaceae } \\
\hline Thelypteris dentata (Forssk.) E.P. St. John & Erva/Terrícola & Naturalizada & Shirasuna 2945 \\
\hline
\end{tabular}

Fanerógamas

Amaranthaceae

Alternanthera brasiliana (L.) Kuntze

Subarbusto/Terrícola

Nativa

Shirasuna 3513

Gomphrena globosa L.

Hebanthe eriantha (Poir.) Pedersen

Subarbusto/Terrícola

Naturalizada

Subarbusto/Terrícola

Nativa

Araceae

Philodendron bipinnatifidum Schott.

Erva/Hemi-epífita

Nativa

Shirasuna 2946

Aristolochiaceae

Aristolochia melastoma Silva Manso ex Duch.

Trepadeira/Terrícola

Nativa

Shirasuna 3515

Asteraceae

Achyrocline satureioides (Lam.) DC.

Erva/Terrícola

Nativa

Shirasuna 2954

Campuloclinum macrocephalum (Less.) DC.

Cyrtocymura scorpioides (Lam.) H.Rob.

Subarbusto/Terrícola

Nativa

Shirasuna 2943

Heterocondylus cf. alatus (Vell.) R.M.King \& H.Rob.

Erva/Terrícola

Nativa

Shirasuna 3517

Subarbusto/Terrícola

Nativa 
Tabela 1 (continuação)

\begin{tabular}{l}
\hline Espécie \\
\hline Mikania cordifolia (L.f.) Willd. \\
Mikania hirsutissima DC. \\
Porophyllum ruderale (Jacq.) Cass. \\
Pterocaulon lanatum Kuntze \\
Senecio adamantinus Bong. \\
Symphyopappus compressus (Gardner) B.L.Rob. \\
Vernonia polyanthes Less. \\
Vernonia sp. \\
Tridax procumbens L. \\
Trixis antimenorrhoea (Schrank) Kuntze \\
Bignoniaceae \\
Amphilophium crucigerum (L.) L.G. Lohmann \\
Pyrostegia venusta (Ker Gawl.) Miers \\
Boraginaceae \\
Cordia polycephala (Lam.) I.M. Jonst. \\
Heliotropium indicum L. \\
Heliotropium lanceolatum Ruiz \& Pav.
\end{tabular}

Bromeliaceae

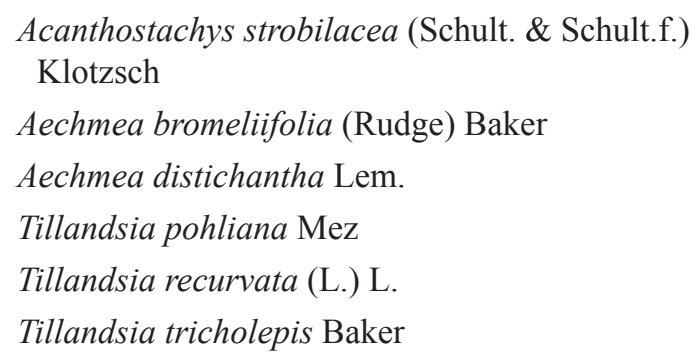

Cactaceae

Epiphyllum phyllanthus (L.) Haw.

Convolvulaceae

Dichondra repens J.R. Forst. \& G. Forst.

Ipomoea hederifolia $\mathrm{L}$.

Ipomoea quamoclit $\mathrm{L}$.

Ipomoea triloba L.

Jacquemontia sp.

Merremia aegyptia (L.) Urb.

Cucurbitaceae

Melothria pendula $\mathrm{L}$.

Momordica charantia L.

Trepadeira/Terrícola Nativa

Trepadeira/Terrícola Nativa

Cyperaceae

Cyperus distans L.

Erva/Terrícola

Nativa

Dioscoreaceae

Dioscorea sp.

Trepadeira/Terrícola

Nativa

Shirasuna 3152 
Tabela 1 (continuação)

\begin{tabular}{|c|c|c|c|}
\hline Espécie & Forma de vida/substrato & Origem & Voucher \\
\hline \multicolumn{4}{|l|}{ Fabaceae } \\
\hline Chamaecrista flexuosa (L.) Greene & Subarbusto/Terrícola & Nativa & \\
\hline Lablab purpureus (L.) Sweet & Trepadeira/Terrícola & Naturalizada & Shirasuna 4072 \\
\hline Macroptilium erythroloma (Mart. ex Benth.) Urb. & Trepadeira/Terrícola & Nativa & \\
\hline \multicolumn{4}{|l|}{ Lamiaceae } \\
\hline Marsypianthes chamaedrys (Vahl) Kuntze & Erva/Terrícola & Nativa & \\
\hline \multicolumn{4}{|l|}{ Malpighiaceae } \\
\hline Amorimia rigida (A. Juss.) W.R. Anderson & Trepadeira/Terrícola & Nativa & Shirasuna 4073 \\
\hline \multicolumn{4}{|l|}{ Malvaceae } \\
\hline Sida rhombifolia L. & Erva/Terrícola & Nativa & \\
\hline Urena lobata $\mathrm{L}$. & Subarbusto/Terrícola & Nativa & Shirasuna 3524 \\
\hline Wissadula hernandioides (L. Hér.) Garcke & Subarbusto/Terrícola & Nativa & Shirasuna 2938 \\
\hline \multicolumn{4}{|l|}{ Melastomataceae } \\
\hline Tibouchina herbacea (DC.) Cogn. & Subarbusto/Terrícola & Nativa & \\
\hline \multicolumn{4}{|l|}{ Menispermaceae } \\
\hline Cissampelos glaberrima A.St.-Hil. & Trepadeira/Terrícola & Nativa & \\
\hline \multicolumn{4}{|l|}{ Orchidaceae } \\
\hline Cyclopogon elatus (Sw.) Schltr. & Erva/Terrícola & Nativa & \\
\hline Catasetum fimbriatum (C. Morren) Lindl. & Erva/Epífita & Nativa & \\
\hline Campylocentrum sp. & Erva/Epífita & Nativa & Shirasuna 4074 \\
\hline Ionopsis utricularioides (Sw.) Lindl. & Erva/Epífita & Nativa & Shirasuna 3525 \\
\hline Oeceoclades maculata (Lindl.) Lindl. & Erva/Terrícola & Naturalizada & \\
\hline \multicolumn{4}{|l|}{ Oxalidaceae } \\
\hline Oxalis cratensis Oliv. ex Hook.** & Erva/Terrícola & Nativa & Shirasuna 3028 \\
\hline \multicolumn{4}{|l|}{ Piperaceae } \\
\hline Piper pseudopothifolium C.DC. & Subarbusto/Terrícola & Nativa & \\
\hline Piper amalago $\mathrm{L}$. & Subarbusto/Terrícola & Nativa & Shirasuna 2940 \\
\hline Piper umbellatum L. & Subarbusto/Terrícola & Nativa & \\
\hline \multicolumn{4}{|l|}{ Poaceae } \\
\hline Axonopus fissifolius (Raddi) Kuhlm. & Erva/Terrícola & Nativa & Shirasuna 3533 \\
\hline Cenchrus ciliaris $\mathrm{L}$. & Erva/Terrícola & Invasora & Shirasuna, 2931 \\
\hline Chloris elata Desv. & Erva/Terrícola & Nativa & Shirasuna 2972 \\
\hline Chloris orthonoton Döll & Erva/Terrícola & Nativa & Shirasuna 2937 \\
\hline Dactyloctenium aegyptium (L.) Willd. & Erva/Terrícola & Naturalizada & Shirasuna 3530 \\
\hline Digitaria insularis (L.) Fedde & Erva/Terrícola & Naturalizada & \\
\hline Digitaria horizontalis Willd. & Erva/Terrícola & Naturalizada & Shirasuna, 2934 \\
\hline Eleusine indica (L.) Gaertn. & Erva/Terrícola & Naturalizada & Shirasuna, 2932 \\
\hline Lasiacis ligulata Hitchc. \& Chase & Erva/Terrícola & Nativa & Shirasuna, 2936 \\
\hline Leptochloa virgata (L.) P. Beauv. & Erva/Terrícola & Nativa & Shirasuna 3529 \\
\hline $\begin{array}{l}\text { Megathyrsus maximus (Jacq.) B.K. Simon \& } \\
\text { S.W.L.Jacobs }\end{array}$ & Subarbusto/Terrícola & Invasora & \\
\hline
\end{tabular}


Tabela 1 (continuação)

\begin{tabular}{|c|c|c|c|}
\hline Espécie & Forma de vida/substrato & Origem & Voucher \\
\hline Melinis repens (Willd.) Zizka & Erva/Terrícola & Invasora & \\
\hline Oplismenus hirtelus subsp. setarius (Lam.)Ekman & Erva/Terrícola & Nativa & $\begin{array}{l}\text { Shirasuna, 2930, } \\
2959\end{array}$ \\
\hline Panicum pilosum $\mathrm{Sw}$. & Erva/Terrícola & Nativa & \\
\hline Panicum sellowii Nees & Erva/Terrícola & Nativa & Shirasuna 2956 \\
\hline Paspalum conjugatum Bergius & Erva/Terrícola & Nativa & $\begin{array}{l}\text { Shirasuna 3532, } \\
2973\end{array}$ \\
\hline Paspalum mandiocanum Trin. & Erva/Terrícola & Nativa & Shirasuna 3534 \\
\hline Paspalum commutatum Nees & Erva/Terrícola & Nativa & Shirasuna 2960 \\
\hline Cenchrus purpureus (Schumach.) Morrone & Erva/Terrícola & Invasora & Shirasuna 2944 \\
\hline Pseudechinolaena polystachya (Kunth) Stapf & Erva/Terrícola & Nativa & \\
\hline Setaria sulcata Raddi & Erva/Terrícola & Nativa & Shirasuna 3531 \\
\hline Sorghum arundinaceum (Desv.) Stapf & Subarbusto/Terrícola & Exótica & Shirasuna, 2935 \\
\hline $\begin{array}{l}\text { Urochloa brizantha (Hochst. ex A. Rich.) } \\
\text { R.D. Webster }\end{array}$ & Erva/Terrícola & Invasora & Shirasuna 2955 \\
\hline Urochloa plantaginea (Link) Webster & Erva/Terrícola & Invasora & Shirasuna 2961 \\
\hline Urochloa mutica (Forssk.) T.Q. Nguyen & Erva/Terrícola & Invasora & Shirasuna 2970 \\
\hline \multicolumn{4}{|l|}{ Rosaceae } \\
\hline Rubus brasiliensis Mart. & Subarbusto/Terrícola & Nativa & \\
\hline \multicolumn{4}{|l|}{ Rubiaceae } \\
\hline Psychotria carthagenensis Jacq. & Subarbusto/Terrícola & Nativa & Shirasuna 3526 \\
\hline \multicolumn{4}{|l|}{ Sapindaceae } \\
\hline Serjania multiflora Cambess. & Trepadeira/Terrícola & Nativa & \\
\hline \multicolumn{4}{|l|}{ Solanaceae } \\
\hline Cestrum mariquitense Kunth & Subarbusto/Terrícola & Nativa & Shirasuna 3538 \\
\hline Nicotiana longiflora $\mathrm{Cav}$ & Erva/Terrícola & Nativa & \\
\hline Solanum sp. & Subarbusto/Terrícola & Nativa & Shirasuna 2957 \\
\hline \multicolumn{4}{|l|}{ Verbenaceae } \\
\hline Lantana camara $\mathrm{L}$. & Subarbusto/Terrícola & Nativa & Shirasuna 3528 \\
\hline Lantana trifolia $\mathrm{L}$. & Subarbusto/Terrícola & Nativa & \\
\hline Stachytarpheta cayennensis (Rich.) Vahl & Erva/Terrícola & Nativa & \\
\hline \multicolumn{4}{|l|}{ Vitaceae } \\
\hline Cissus verticillata (L.) Nicolson \& C.E.Jarvis & Trepadeira/Terrícola & Nativa & Shirasuna 2948 \\
\hline
\end{tabular}

a riqueza de espécies menor em áreas com maior intervenção. Desse modo, sabe-se que a riqueza de espécies epífitas em áreas secundárias é muito inferior àquela registrada em florestas maduras (Barthlott et al. 2001).

Algumas espécies podem estar associadas ao grau de intervenção antrópica, sendo mais abundantes em áreas com maior intensidade de degradação, como no caso de algumas espécies de trepadeiras e ervas terrícolas do grupo das gramíneas (Engel et al. 1998, Fine 2002, Martins et al. 2004). Neste trabalho foram registradas duas espécies que se comportam como invasoras: Megathyrsus maximus (Jacq.) B.K. Simon \& S.W.L. Jacobs e Urochloa brizantha (Hochst. ex A. Rich.) R.D. Webster, que se mantêm em determinadas áreas do sub-bosque, principalmente em locais com maior abertura do dossel. Espécies como Cenchrus ciliares L., Cenchrus purpureus 
(Schumach.) Morrone, Dactyloctenium aegyptium (L.) Willd., Eleusine indica (L.) Gaertn., Melinis repens (Willd.) Zizka e Sorghum arundinaceum (Desv.) Stapf foram encontradas nas margens do reflorestamento, avançando alguns metros da borda para o interior da área.

No entanto, observa-se que a ocorrência de outras espécies de ervas, trepadeiras e subarbustos podem estar associadas a melhores condições de conservação, ou fazem parte de estratégias regenerativas do processo sucessional (Engel et al. 1998, Schnitzer \& Bongers 2002). Na RPPN foram encontradas espécies típicas de sub-bosque que ocorreram em áreas mais conservadas, em condições de sombreamento, tais como as seguintes espécies de Poaceae: Lasiacis ligulata Hitchc. \& Chase, erva típica de sub-bosque que apresenta sementes zoocóricas; Oplismenus hirtelus subsp. setarius (Lam.) Ekman, Pseudechinolaena polystachya (Kunth) Stapf, Panicum pilosum Sw. e Panicum sellowii Nees, ervas que formam grupamentos, comportando-se como forrageiras no solo do sub-bosque; e Paspalum mandiocanum Trin. e Setaria sulcata Raddi, ervas que se encontraram espalhadas pelo sub-bosque. Trepadeiras como Aristolochia melastoma Silva Manso ex Duch. (Aristolochiaceae), Mikania cordifolia (L.f.) Willd. e Mikania hirsutissima DC. (Asteraceae), amplamente distribuídas no sub-bosque, e Amphilophium crucigerum (L.) L.G. Lohmann e Pyrostegia venusta (Ker Gawl.) Miers (Bignoniaceae), conhecidas como pente-de-macaco e erva-de-são-joão, respectivamente, que procuram a copa das árvores em busca de maior intensidade luminosa. Amorimia rigida (A. Juss.) W.R. Anderson (Malpighiaceae), conhecida como timbó ou tingui com flores amarelas, considerada tóxica para a pecuária (Matos et al. 2011), foi encontrada sobre a copa das árvores. Cissampelos glaberrima A.St.-Hil. (Menispermaceae), trepadeira conhecida como parreira-brava, Serjania multiflora Cambess. (Sapindaceae), trepadeira conhecida como cipó-timbó, e Cissus verticillata (L.) Nicolson \& C.E. Jarvis, conhecida como anil-trepador com frutos zoocóricos, foram encontradas desenvolvendo-se sobre a vegetação arbórea.

Asteraceae, Boraginaceae, Piperaceae, Rubiaceae, Sapindaceae, Solanaceae e Verbenaceae contribuíram com espécies típicas para a formação do estrato herbáceo (ervas) e subarbustivo.

De modo geral, a riqueza de ervas terrícolas e epífitas, subarbustos e trepadeiras na RPPN é superior à riqueza de espécies arbóreas (figura 2). A importância dessas formas de vida para a diversidade local pode ir além da riqueza encontrada na área. Em todo o reflorestamento da RPPN foram observadas e identificadas 15 espécies epífitas e hemi-epífitas, compostas por cinco famílias e 10 gêneros. A riqueza de epífitas encontrada na RPPN evidencia que áreas com reflorestamentos recentes podem ser utilizadas para realocação de determinadas espécies não arbóreas, incluindo epífitas, conforme indagado por Duarte \& Gandolfi (2013). No entanto, a escolha dos forófitos deve ser feita com cautela, evitando árvores pioneiras, por exemplo, uma vez que apresentam ciclo de vida curto (Garcia 2012). Embora seja um reflorestamento com apenas nove anos, a RPPN já foi capaz de fornecer um habitat com condições adequadas de sombreamento para o desenvolvimento dessas espécies.

Vale ainda ressaltar a importância da presença de remanescentes naturais que atuam como fontes de propágulo e que desempenham um papel fundamental no enriquecimento de áreas em processo de restauração, conforme já destacado por diversos autores (Guariguata \& Ostertag 2001, Chazdon et al. 2003, Rodrigues et al. 2011). Nesse contexto, verificou-se que as espécies não arbóreas têm tido meios suficientes para chegar à RPPN, fato já reportado para espécies arbóreas na mesma área de estudo (Silveira et al. 2011, Colmanetti 2013, Trevelin et al. 2013).

As epífitas estabelecem-se em troncos e galhos de forófitos e parecem estar diretamente relacionadas a fatores abióticos como luz, umidade, substrato, e com o estágio sucessional da floresta (Benzing 1990, Fontoura 2001, Kersten \& Kuniyoshi 2009). $\mathrm{O}$ fato das epífitas apresentarem crescimento lento e alta sensibilidade às variações climáticas, em geral,

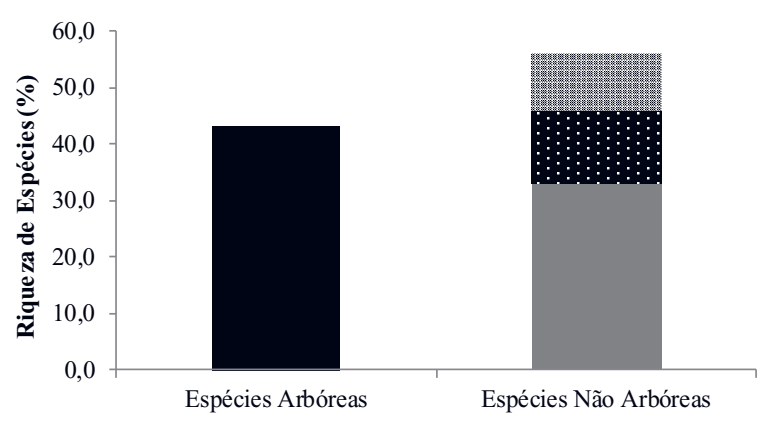

Figura 2. Espécies arbóreas e não arbóreas encontradas na RPPN Parque São Marcelo, Mogi Guaçu, SP, Brasil. « Trepadeiras, - Subarbustos, Ervas.

Figure 2. Woody species and non-woody species present at Parque São Marcelo RPPN, Mogi Guaçu, São Paulo State, Brazil. « Climbers, a Shrubs, [ Herbs. 
torna-as mais vulneráveis, sendo a sua ocorrência um importante indicativo de florestas mais conservadas e restauradas (Hietz 1999). De modo geral, é possível que a presença das epífitas possa refletir o grau de preservação local, uma vez que alguns grupos são menos tolerantes às variações ambientais decorrentes de intervenções antrópicas; destacando-se que a sua ocorrência em florestas secundárias ou reflorestamentos pode ser muito inferior, quando comparada a florestas maduras (Barthlott et al. 2001).

Um aspecto importante do uso da alta diversidade de espécies em reflorestamentos consiste no quesito da maior diversidade de forófitos disponíveis para serem utilizados pelas epífitas como suporte. Considerando que algumas espécies epifíticas têm preferência por determinados tipos de troncos como suporte, incluindo característica como a rugosidade da superfície do tronco (Callaway et al. 2002, Duarte \& Gandolfi 2013), quando se aumenta a diversidade de espécies plantadas, há maior variedade de tipos de troncos disponíveis para serem utilizados como suporte. Dessa forma, espera-se que a alta diversidade e riqueza de espécies utilizadas em reflorestamentos possam favorecer a maior variabilidade de forófitos para as epífitas.

Acredita-se que diversos aspectos, como a umidade e luz, não abordados no presente estudo, podem influenciar a ocorrência e distribuição de espécies não arbóreas. Espera-se que a riqueza, diversidade e distribuição dessas espécies sejam temas de estudos posteriores, considerando sua importância como potencial indicador para monitoramento, uma vez que ainda não é exigida em documentos formais (SMA 32/2014).

Nessa perspectiva, no que tange à Resolução SMA 32/2014, vale evidenciar que se as espécies não arbóreas fossem consideradas como indicadores, sendo incluídas como regenerantes, o número de espécies desse estrato poderia dobrar a riqueza da área em restauração, tendo esse estudo como exemplo, indicando que os Valores Intermediários de Referência exigidos na Resolução deveriam ser reavaliados. Contudo, vale salientar que ainda são necessários mais estudos, principalmente no que se refere à padronização do sistema de amostragem, para que essas espécies possam ser utilizadas como indicadoras.

Embora os estudos ainda sejam iniciais, já foi possível constatar elevada riqueza de espécies não arbóreas, predominantemente nativas, que surgiram por regeneração natural, em número superior à riqueza de espécies arbóreas. A presença de Oxalis cratensis (Oxalidade), considerada ameaçada de extinção, e de
Pellaea viridis (Pteridaceae) referida como segundo registro para o Estado de São Paulo, conferem grande importância dessas formas de vida para a conservação da biodiversidade. O reduzido número de estudos com enfoque na vegetação não arbórea talvez possa ser atribuído à dificuldade de identificação das espécies que os compõem; ou até a pouca atenção que se dá a esses grupos de espécies, um desafio a ser superado, quando se trata de monitoramento de áreas em processo de restauração.

\section{Agradecimentos}

Ao Conselho Nacional de Desenvolvimento Científico e Tecnológico. Ao Programa de PósGraduação em Biodiversidade Vegetal e Meio Ambiente, e ao Instituto de Botânica de São Paulo. À International Paper, e aos seus funcionários Miguel Magela e João Machado, pelo apoio logístico. À Coordenação Especial de Restauração de Áreas Degradadas e aos funcionários da Campininha, pelo apoio em campo. Ao Dr. Pedro Bond Schwaztburg, pela identificação das pteridófitas. À Mayara Pastore, pela identificação das Asteraceae. À Elenice Eliana Teixeira, pela revisão do manuscrito. Aos revisores que contribuíram consideravelmente para melhoria deste manuscrito.

\section{Literatura citada}

APG III. 2009. An update of the Angiosperm Phylogeny Group classification for the orders and families of flowering plants: APG III. Botanical Journal of the Linnean Society 161: 105-121.

Aquino, C. \& Barbosa, L.M. 2009. Classes sucessionais e síndromes de dispersão de espécies arbóreas e arbustivas existentes em vegetação ciliar remanescente (Conchal, $\mathrm{SP}$ ), como subsídio para avaliar o potencial do fragmento como fonte de propágulos para enriquecimento de áreas revegetadas no rio Mogi-Guaçu, SP. Revista Árvore 33: 349-358.

Barthlott, W., Schmit-Neuerburg, V., Nieder, J. \& Engwald, S. 2001. Diversity and abundance of vascular epiphytes: a comparison of secondary vegetation and primary montane rain forest in the Venezuelan Andes. Plant Ecology 152: 145-156.

Bellotto, A., Viani, R.A.G., Nave, A.G., Gandolfi, S. \& Rodrigues, R.R. 2009. Monitoramento das áreas restauradas como ferramenta para avaliação da efetividade das ações de restauração e para redefinição metodológica. In: R.R. Rodrigues, P.H.S. Brancalion \& I. Isernhagen. (orgs.). Pacto pela restauração da mata atlântica: Referencial dos conceitos e ações de restauração florestal. 1 ed. LERF/ESALQ: Instituto BioAtlântica, São Paulo. 
Benzing, D.H. 1990. Vascular epiphytes: general biology and related biota Cambridge University Press. Cambridge, UK.

Callaway, R.M., Reinhart, K.O., Moore, G.W., Moore, W.J. \& Pennings, S.C. 2002. Epiphyte host preferences and host traits: mechanisms for species-specific interactions. Oecologia 132: 221-230.

Chazdon, R.L. 2003. Tropical forest recovery: legacies of human impact and natural disturbances. Perspectives in Plant Ecology, Evolution and Systematics 6: 51-71.

Colmanetti, M.A.A. 2013. Estrutura da vegetação e características edáficas de um reflorestamento com espécies nativas. Dissertação de Mestrado, Instituto de Botânica, São Paulo.

Damasceno, A.C.F. 2005. Macrofauna edáfica, regeneração natural de espécies arbóreas, lianas e epífitas em florestas em processo de restauração com diferentes idades no pontal do Paranapanema. Dissertação de Mestrado, Universidade de São Paulo, Piracicaba.

Dinato, D.O. 2014. Florística vascular não arbórea de um fragmento de Floresta Estacional Semidecidual no município de Analândia - SP. Dissertação de Mestrado, Universidade Estadual Paulista, Rio Claro.

Duarte, M.M. \& Gandolfi, S. 2013. Enriquecimento de florestas em processo de restauração: aspectos de epífitas e forófitos que podem ser considerados. Hoehnea 40: 507-514.

Durigan, G., Engel, V.L. Torezan, J.M., Melo, A.C.G., Marques, M.C.M., Martins, S.V., Reis, A. \& Scarano, F.R. 2010. Normas jurídicas para a restauração ecológica: uma barreira a mais a dificultar o êxito das iniciativas? Revista Árvore 34: 471-485.

Engel, V.L., Fonseca, R.B., Oliveira, R.D. 1998. Ecologia de lianas eo manejo de fragmentos florestais. Série técnica IPEF 12: 43-64.

Filgueiras, T.S., Brochado, A.L., Nogueira, P.E., \& Gualla Ii, G.F. 1994. Caminhamento - Um método expedito para levantamentos florísticos qualitativos. Caderno de Geociência IBGE 12: 39-43.

Fine, P.V. 2002. The invasibility of tropical forests by exotic plants. Journal of Tropical Ecology 18: 687-705.

Fontoura, T. 2001. Bromeliaceae e outras epífitasestratificação e recursos disponíveis para animais na Reserva Ecológica Estadual de Jacarepiá, Rio de Janeiro. Bromélia 6: 33-39.

Flora do Brasil. 2015. Lista de Espécies da Flora do Brasil. Jardim Botânico do Rio de Janeiro. Disponível em http:// floradobrasil.jbrj.gov.br/ (acesso em 01-VII-2015).

Garcia, L.C. 2012. Avaliação da sustentabilidade ecológica de matas ciliares em processo de restauração. Tese de doutorado, Universidade Estadual de Campinas, Campinas.

Guariguata, M.R. \& Ostertag, R. 2001. Neotropical secondary forest succession: changes in structural and functional characteristics. Forest Ecology and Management 148: 185-206.
Hietz, P. 1999. Diversity and conservation of epiphytes in a changing environment. Pure Appl. Chem 70: 2114-2125.

IBGE. 2012. Instituto Brasileiro de Geografia e Estatística. Manual técnico da vegetação brasileira. Manuais técnicos em geociências.

Kersten, R. \& Kuniyoshi, Y. S. 2009. Conservação das florestas na bacia do alto Iguaçu, Paraná - Avaliação da comunidade de epífitas vasculares em diferentes estágios serais. Floresta 39: 51-66.

Köopen, W. 1948. Climatologia. Fondo de Cultura Económica, México.

Martins, C.R., Leite, L.L. \& Haridasan, M. 2004. Capim-gordura (Melinis minutiflora P. Beauv.), uma gramínea exótica que compromete a recuperação de áreas degradadas em unidades de conservação. Revista Árvore 28: 739-747.

Matos, F.J.A., Lorenzi, H., Santos, L.F.L., Matos, M.E.O., Silva, M.G.V., \& Souza, M.P. 2011. Plantas tóxicas. Estudos de fitotoxicologia química e plantas brasileiras. Instituto Plantarum de Estudos da Flora, Nova Odessa.

Melo, A.C.G.D., Miranda, D.L.C.D. \& Durigan, G. 2007. Cobertura de copas como indicador de desenvolvimento estrutural de reflorestamentos de restauração de matas ciliares no Médio Vale do Paranapanema, SP, Brasil. Revista Árvore 31: 321.

Mamede, M.C.H., Souza, V.C., Prado, J., Barros, F., Wanderley, M.G.L. \& Rando, J.G. 2007. Livro vermelho das espécies vegetais ameaçadas de extinção no Estado de São Paulo. Imprensa Oficial, São Paulo.

Menini Neto, L., Matozinhos, C.N., Abreu, N.L., Valente, A.S.M., Antunes, K., Souza, F.S., Viana, P.L., \& Salimena, F.R.G. 2009. Flora vascular não-arbórea de uma floresta de grota na Serra da Mantiqueira, Zona da Mata de Minas Gerais, Brasil. Biota Neotropica 9: $149-161$.

Mobot. 2015. Missouri Botanical Garden Plant Science. Database - W3 TRÓPICOS. Disponível em http://www. mobot.org (acesso em 01-IV-2015).

Mori, S.A., Silva, L.A.M., Lisboa, G. \& Coradin, L. 1985. Manual de Manejo do Herbário Fanerogâmico. CEPEC, Ilhéus.

Moro, M.F., Souza, V.C., Oliveira-Filho, A.T., Queiróz, L.P., Fraga, C.N., Rodal, M.J.N., Araújo, F.S. \& Martins, F.R. 2012. Alienígenas na sala: o que fazer com espécies exóticas em trabalhos de taxonomia, florística e fitossociologia? Acta Botanica Brasilica 26: 991-999.

Oliveira, A.P.C. 2012. Flora vascular não-arbórea do Parque Estadual De Porto Ferreira, SP, Brasil. Dissertação de Mestrado, Universidade Estadual Paulista, Rio Claro.

Prado, J. \& Hirai, R.Y. 2011. Pellaea flavescens Fée in Rio de Janeiro, its Lectotypification, and its New Record for São Paulo State, Brazil. American Fern Journal 101: 50-56. 
Prado, J. 2015. Pteridaceae. In: Lista de Espécies da Flora do Brasil. Jardim Botânico do Rio de Janeiro. Disponível em http://www.floradobrasil.jbrj.gov.br/ jabot/floradobrasil/FB130943 (acesso em 22-IX-2015).

Rodrigues, R.R., Gandolfi, S., Nave, A.G.; Aronson, J., Barreto, T.E., Vidal C.Y. \& Brancalion, P.H.S. 2011. Large-scale ecological restoration of high-diversity tropical forests in SE Brazil. Forest Ecology and Management 261: 1605-1613.

Rossatto, D.R., Toniato, M.T., Zugliani \& Durigan, G. 2008. Flora fanerogâmica não-arbórea do cerrado na Estação Ecológica de Assis, Estado de São Paulo. Revista Brasileira de Botânica 31: 409-424.

São Paulo. 2014. Resolução SMA No 32, de 03 de abril de 2014 estabelece as orientações, diretrizes e critérios sobre restauração ecológica no Estado de São Paulo, e dá providências correlatas. Diário Oficial do Estado de São Paulo - Meio Ambiente.

Schnitzer, S.A. \& Bongers, F. 2002. The ecology of lianas and their role in forests. Trends in Ecology \& Evolution 17: 223-230.

Silveira, M., Trevelin, L., Port-Carvalho, M., Godoi, S., Mandetta, E.N. \& Cruz-Neto, A.P. 2011. Frugivory by phyllostomid bats (Mammalia: Chiroptera) in a restored area in Southeast Brazil. Acta Oecologica 37: 31-36.
Smith, A.R., Pryer, K.M., Schuettpelz, E., Korall, P., Schneider, H. \& Wolf, P.G. 2006. A classification for extant ferns. Taxon 55: 705-731.

Siqueira, L.P. 2002. Monitoramento de áreas restauradas no interior do estado de São Paulo, Brasil. Dissertação de Mestrado, Universidade de São Paulo, Piracicaba.

Sorreano, M.C.M. 2002. Avaliação de aspectos da dinâmica de florestas restauradas, com diferentes idades. Dissertação de Mestrado, Universidade de São Paulo, Piracicaba.

Souza, F.M. \& Batista, J.L.F. 2004. Restoration of seasonal semideciduous forests in Brazil: influence of age and restoration design on forest structure. Forest Ecology and Management 191: 185-200.

Suganuma, M.S., Torezan, J.M.D., Cavalheiro, A.L., Vanzela, A.L.L. \& Benato, T. 2008. Comparando metodologias para avaliar a cobertura do dossel ea luminosidade no sub-bosque de um reflorestamento e uma floresta madura. Revista Árvore 32: 377-385.

Trevelin, L.C., Silveira, M., Port-Carvalho, C., Homem, D.H. \& Cruz-Neto, A.P. 2013. Use of space by frugivorous bats (Chiroptera: Phyllostomidae) in a restored Atlantic forest fragment in Brazil. Forest Ecology and Management 291: 136-143.

Zenni, R.D. \& Ziller, S.R. 2011. An overview of invasive plants in Brazil. Revista Brasil de Botânica. 34: 431-446. 